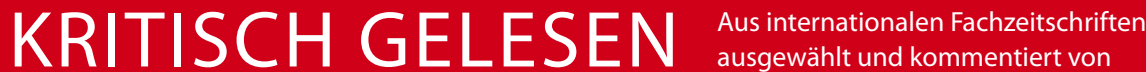

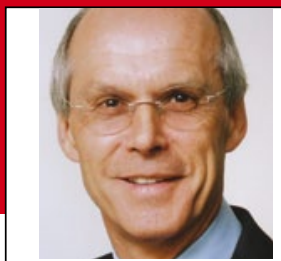

Prof. Dr. med. H. S. FüeßI

Isar-Amper-

Klinikum,

KI. München-Ost,

Haar

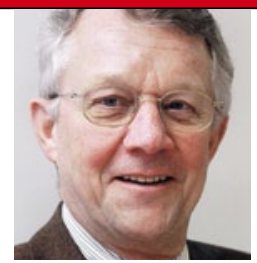

Prof. Dr. med. H. Holzgreve

Internist,

Kardiologische

Praxis, München

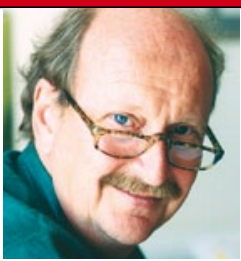

Prof. Dr. med.

E. Ernst

Peninsular

Medical School,

University

of Exeter/UK

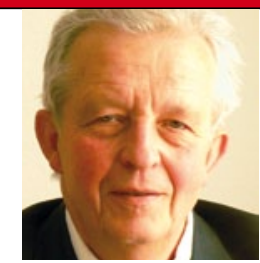

Prof. Dr. med. K. Malberg Immunologie, DresdenLoschwitz

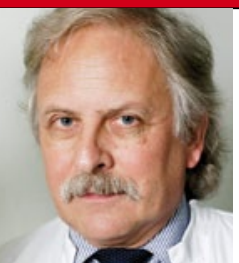

Prof. Dr. med. H.-C. Diener

Klinik für

Neurologie,

Universitätsklinik Essen

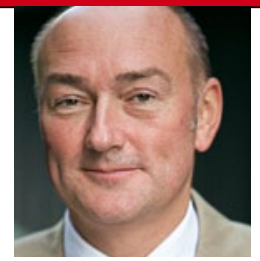

PD Dr. med. W. Kleophas

Gemeinschaftspraxis Düsseldorf

\section{Mit süßer Verführung gegen Herztod und Schlaganfall}

\begin{abstract}
Viele Menschen lieben Schokolade, scheuen aber Gewichtszunahme und nachteilige gesundheitliche Folgen. Doch vieles spricht dafür, dass Schokolade vor kardiovaskulären Erkrankungen schützt.
\end{abstract}

— Nach einer gründlichen Recherche fanden die Autoren sieben Studien mit 114009 Probanden, die den Einfluss von

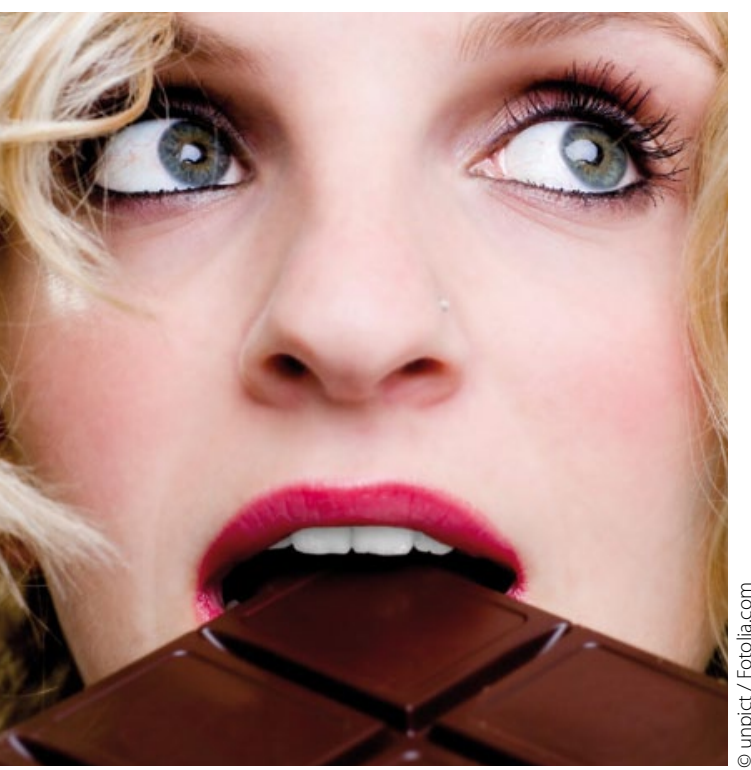

Sollte es tatsächlich etwas geben, was Spaß macht und auch noch gesund ist?
Schokoladenverzehr auf die Häufigkeit kardiovaskulärer Erkrankungen überprüften. Die Beobachtungsdauer lag zwischen acht und 16 Jahren.

Bei höherem Konsum von Schokolade lag das Risiko für alle kardiovaskulären Erkrankungen um 37\% ( $\mathrm{P}=$ 0,001), für Schlaganfälle um $29 \%$ und für Herzinsuffizienz um 5\% niedriger. Insgesamt wurde die Korrelation von Konsum zu Erkrankungshäufigkeit anhand von 13 Kriterien überprüft. Zwölfmal erwies sich Schokolade als protektiv. Nur eine Studie ergab einen ungünstigen Effekt auf das Risiko für Herzinsuffizienz. Nach Daten aus Japan sinkt das Diabetesrisiko durch hohen Schokoladenkonsum bei Männern und Frauen um 35 bzw. 27\%.

\section{Kommentar}

Die Probandenzahl ist zwar beachtlich, allerdings sind die Methoden der Studien nicht von höchster Qualität (sechs Kohorten- und eine Querschnittsstudie), auch wurden der Schokoladenkonsum und die Endpunkte unterschiedlich definiert. Doch das Ergebnis ist für den Endpunkt aller kardiovaskulären Erkrankungen signifikant und liegt für Schlaganfälle und Herzinsuffizienz im positiven Trend. Häufig wird zwar vor-

\begin{tabular}{l|l|l}
\hline Tabelle 1 & & \\
Endpunkt & $\begin{array}{l}\text { Risiko- } \\
\text { reduktion }\end{array}$ & $\begin{array}{l}\text { P- } \\
\text { Wert }\end{array}$ \\
\hline $\begin{array}{l}\text { Alle KV } \\
\text { Erkrankungen (6) }\end{array}$ & $-37 \%$ & 0,001 \\
\hline $\begin{array}{l}\text { Schlaganfall } \\
\text { (3) }\end{array}$ & $-29 \%$ & ns \\
\hline $\begin{array}{l}\text { Herzinsuffizienz } \\
\text { (2) }\end{array}$ & $-5 \%$ & ns \\
\hline $\begin{array}{l}\text { Abnahme von kardiovaskulären Er- } \\
\text { krankungen bei hohem gegenüber } \\
\text { geringem Schokoladenkonsum (in } \\
\text { Klammern: Zahl der Studien; ns = } \\
\text { nicht signifikant). }\end{array}$ \\
\hline
\end{tabular}

zugsweise dunkle Schokolade propagiert, doch in dieser Analyse wurden alle Schokoladensorten berücksichtigt. In zahlreichen klinischen und experimentellen Studien wurden antioxidative, antiinflammatorische, antiatherogene, antithrombotische und antihypertensvie Wirkungen der Schokolade ebenso nachgewiesen wie günstige Einflüsse auf Insulinsensitivität, vaskuläre Endothelfunktion und Aktivierung von Stickstoffoxyd. Als wirksames Agens gelten die Flavonoide. Während gegen reichlich Essen, Trinken und Rauchen Bedenken bestehen, wäre der Verzehr von Schokolade die erste Maßnahme, die gleichzeitig Spaß macht und die Gesundheit fördert.

H. HolzGREVE =

- A. Buitrago-Lopez et al.

Chocolate consumption and cardiometabolic disorders: systematic review and meta-analysis. Brit. Med. J. 343 (2011) 343:4488 